\title{
Subspace-Based Blind and Group-Blind Space-Time Multiuser Detection for the Generalized Multicarrier DS-CDMA Uplink
}

\author{
Bin Hu, Lie-Liang Yang and Lajos Hanzo \\ School of ECS, University of Southampton, SO17 1BJ, UK. \\ Tel: +44-23-8059 3125, Fax: +44-23-8059 4508 \\ Email: bh202r,lly,lh@ecs.soton.ac.uk; http://www-mobile.ecs.soton.ac.uk
}

\begin{abstract}
Subspace-based blind and group-blind space-time multiuser detection is invoked for a smart antenna aided generalized MC DS-CDMA system communicating over a dispersive Space-Time (ST) Rayleigh fading channel, where the channel estimates are attained by using subspacebased blind techniques. In the generalized MC DS-CDMA system considered, smart antennas are employed, in order to provide increased degrees of freedom and hence to improve the attainable performance and user capacity. Furthermore, our simulation results confirm that in the proposed multiple antenna aided generalized MC DS-CDMA system, the blind and group-blind space-time multiuser detectors equipped with the subspacebased blind channel estimator are capable of achieving a similar performance to that exploiting the perfect knowledge of the channel.
\end{abstract}

\section{INTRODUCTION}

Recently, considerable amount of research has been devoted to Multi-User Detection (MUD) [2], [3], [4], [5], [1], which is capable of essentially eliminating the effects of multiuser interference and hence has the potential of substantially increasing the capacity of CDMA systems. Verdu's optimum MUD presented in [2] is capable of attaining a near-single-user performance at the expense of an exponentially increasing complexity imposed by increasing the number of users supported. By contrast, a decorrelating MUD having a lower complexity was proposed in [6], where a slight increase of the achievable BER was incurred. Training sequence based adaptive MUD was introduced in [7], [8], [9], which requires the knowledge of the signature waveform and the timing information of the desired user, but no other knowledge is necessitated. In contrast to training sequence based adaptive MUD, the Minimum-Output-Energy (MOE) based blind adaptive MUD [3] does not require any training sequence and hence achieves an increased spectrum efficiency, although its performance is suboptimum in the high-SNR region. In [4], [10], Wang and Poor proposed a novel blind MUD for single-carrier DS-CDMA systems based on the philosophy of signal subspace estimation, which benefits from a lower computational complexity and a better performance compared to the MOE based blind MUD. Group-blind MUDs having the prior knowledge of all signature waveforms of the intracell users were then proposed in [5] for the uplink of a single-carrier DS-CDMA system, which exhibited a significant performance improvement over that of the blind MUDs advocated in [4], [10]. Furthermore, in [11], a subspace-based MMSE receiver was proposed for a Multi-Carrier Direct Sequence Code Division Multiple Access (MC DS-CDMA) system, where the orthogonality between the noise subspace and the desired signal vector was exploited for blindly extracting both the required timing and channel information.

In this contribution, subspace-based blind and group-blind spacetime MUDs are invoked as joint space-time-frequency domain MUDs

The financial support of the European Union under the auspices of the Phoenix and Newcom projects and that of the EPSRC is gratefully acknowledged. for the smart antenna aided generalized MC DS-CDMA system of [12], since subspace-based estimation techniques [4], [10], [11] are capable of extracting the channel estimates required. More explicitly, the novelty of this contribution is that we combine the benefits of subspacebased blind and group-Blind space-time MUDs with those of the smart antenna aided generalized MC DS-CDMA system of [12] for the sake of improving the achievable performance of the system by jointly performing multiuser detection in the space-time-frequency domain, while achieving both frequency and spatial diversity.

The rest of this paper has the following structure. In Section II the philosophy of the generalized MC DS-CDMA system employing smart antennas will be described and characterized. Then, a subspacebased blind channel estimation technique is invoked for the smart antenna aided generalized MC DS-CDMA system considered in Section III. Section IV develops subspace-based blind and group-blind spacetime MUDs in the context of a smart antenna aided generalized MC DS-CDMA system. The attainable performance of these MUDs is studied comparatively in Section V. Finally, we provide our conclusions in Section VI.

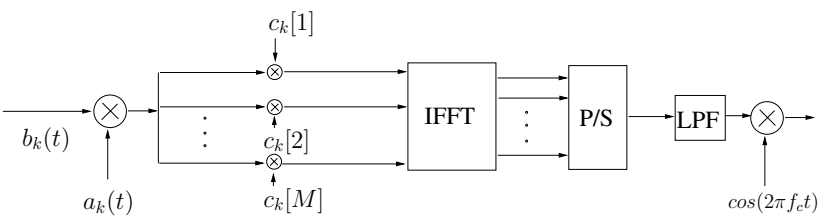

Fig. 1. Transmitter schematic of MC DS-CDMA using both $N$-chip timedomain and $V$-chip frequency-domain spreading

\section{SySTEM MODEL}

The transmitter of the generalized MC DS-CDMA system is portrayed in Fig.1. At the transmitter side, the binary data stream $b_{k}(t)$ having a bit duration of $T_{b}$ is spread using an $N$-chip Time Domain (TD) DS spreading waveform $c_{k}(t)$ and the chip duration was $T_{c}=T_{b} / N$. The DS spread signals are simultaneously modulated using BPSK and then spread using an orthogonal Frequency Domain (FD) spreading sequence $\mathbf{c}^{\prime}{ }_{k}=\left[c^{\prime}{ }_{k, 0}, c^{\prime}{ }_{k, 1}, \ldots, c^{\prime}{ }_{k, V-1}\right]$ of length $V$, where we have $\mathbf{c}^{\prime}{ }_{k} \cdot \mathbf{c}^{\prime H}{ }_{k}=1$. In this section we assume that OFDM using $V$ subcarriers was invoked [13], where $V$ consecutive chips of the MC DS-CDMA spreading sequences were mapped to $V$ different subcarriers during an OFDM symbol and hence the OFDM-chip duration was $T_{c^{\prime}}=\frac{T_{b}}{N V}$. The spread OFDM chip-vector of $V$ subcarriers can be expressed as $\mathbf{s}_{k}(t)=\mathbf{c}^{\prime}{ }_{k} b_{k}(t) c_{k}(t)=\left[s_{1}(t), s_{2}(t), \ldots, s_{V}(t)\right]$. The Inverse Fast Fourier Transform (IFFT) is then invoked for modulating the $V$ subcarriers by using the spread OFDM chip-vector $\mathbf{s}_{k}(t)$ [13]. The output signal of the IFFT-based demodulator is a block of $V$ number of time domain samples in parallel form. After Parallel to 
Serial (P/S) conversion, a sufficiently long cyclic prefix is inserted in the OFDM symbol for the sake of compensating for the delay-spreadinduced Inter-Symbol Interference (ISI) imposed by the dispersive channel [13]. Then, these time domain signals are transmitted through a multipath fading channel, which is assumed to have $L$ paths for all the $K$ users. Upon assuming that at the Base-Station (BS) there are $M$ number of receiver antenna elements and the channel encountered is time-invariant during three consecutive bits, the Spatio-Temporal (ST) Channel Impulse Response (CIR) $h_{k m}(t)$ between the $k$ th user and the $m$ th Antenna Element (AE) can be expressed as

$$
h_{k m}(t)=\sum_{l=0}^{L-1} h_{k m, l} \delta\left(t-l T_{c^{\prime}}\right)
$$

where $h_{k m, l}$ is the complex-valued channel gain experienced by the signal of the $k$ th user in the $l$ th path impinging on the $m$ th AE, which obeys Rayleigh fading.

At each AE, the time domain samples of the received signal corresponding to the cyclic prefix are first removed and $V$-point Fast Fourier Transform (FFT) is invoked for demodulating the remaining $V$ samples and for generating the demodulated subcarrier signals in the frequency domain [13]. Consequently, the received signal can be written as $\mathbf{r}(t)=\left[\mathbf{r}_{0}^{T}(t), \mathbf{r}_{1}^{T}(t), \ldots, \mathbf{r}_{M-1}^{T}(t)\right]^{T}$, where $\mathbf{r}_{m}(t) \in \mathcal{R}^{V}$ is given by

$$
\begin{aligned}
\mathbf{r}_{m}(t)= & \sum_{k=1}^{K} \mathbf{C}_{k}^{\prime} \mathbf{H}_{k m} \sum_{i=0}^{M_{F}-1} A_{k} b_{k}[i] \mathbf{c}_{k}\left(t-i T_{b}-\tau_{k}\right) \\
& +\mathbf{n}_{m}(t)
\end{aligned}
$$

and $\mathbf{H}_{k m}=\left[H_{k m, 0}, H_{k m, 1}, \ldots, H_{k m, V-1}\right]^{T}$ denotes the Frequency-Domain Channel Transfer Function (FDCTF) between the $k$ th user and the $m$ th AE. In (2), $M_{F}$ is the length of the transmitted data frame, while $A_{k},\left\{b_{k}[i]\right\}$ and $0 \leq \tau_{k}<T_{b}$ represent the received signal amplitude, symbol stream and the delay of the $k$ th user, respectively. The spreading sequence $\mathbf{c}_{k}(t)=\sum_{j=0}^{N-1} c_{k}(j) \psi\left(t-j T_{c}\right)$ in (2) denotes the signature waveform of the $k$ th user, where $c_{k}(j)$ assumes values of +1 or -1 with equal probability, while $\psi(t)$ is a normalized chip waveform of duration $T_{c}$. Furthermore, in (2), $\mathbf{C}^{\prime}{ }_{k}=\operatorname{diag}\left\{\mathbf{c}^{\prime}{ }_{k}\right\}$ and $\mathbf{n}_{m}(t)$ represents the AWGN vector associated with the covariance matrix of $\sigma^{2} \mathbf{I}_{V}$, where $\mathbf{I}_{V}$ denotes the $(V \times V)$-dimensional identity matrix. Since we assume that a sufficiently long cyclic prefix was inserted and we have $V \geq L$, every subcarrier experiences flat fading and hence no OFDM ISI is incurred. Therefore, the FDCTF $\mathbf{H}_{k m}$ in (2) can be expressed as the $V$-point DFT of $\mathbf{h}_{k m}=\left[h_{k m, 0}, \ldots, h_{k m, L-1}\right]^{T}$ in the form of $\mathbf{H}_{k m}=\mathbf{F}_{L} \cdot \mathbf{h}_{k m}$, where $\mathbf{F}_{L}$ is an $(V \times L)$-dimensional matrix, which is given by the first $L$ columns of the DFT matrix $\mathbf{F}$ formulated as $\mathbf{F}=\left(\begin{array}{cccc}1 & 1 & \cdots & 1 \\ 1 & e^{-j 2 \pi / V} & \cdots & e^{-j 2 \pi(V-1) / V} \\ \vdots & \vdots & \ddots & \vdots \\ 1 & e^{-j 2 \pi(V-1) / V} & \cdots & e^{-j 2 \pi(V-1)(V-1) / V}\end{array}\right)$.

A set of $M \cdot V$ chip-matched filters are used for sampling the received signal $\mathbf{r}(t)$ at a rate of $1 / T_{c}$. Since the delay spread is often assumed to be one symbol interval of $T_{b}$ duration in practical CDMA systems [10], we observe the chip-matched filter outputs for a duration of $2 T_{b}$ so that despite the channel-induced ISI a completely interference-free symbol of the desired user is guaranteed to be observed. Hence the $n$th received signal sample of the $v$ th chip-matched filter corresponding to the $m$ th AE during the $l$ th symbol has the form of

$$
r_{m, v}[l, n]=r_{m, v}\left(l T_{b}+n T_{c}\right)=\sum_{k=1}^{K} y_{k m, v}[l, n]+n_{m, v}[l, n],
$$

where $n_{m, v}[l, n]$ is the AWGN component and

$$
\begin{aligned}
y_{k m, v}[l, n]= & A_{k} H_{k m, v} c^{\prime}{ }_{k, v} \sum_{i=0}^{M_{F}-1} \sum_{j=0}^{N-1} b_{k}[i] c_{k}(j) \\
& \times \hat{\psi}\left((l N+n-j-i N) T_{c}-\tau_{k}\right)
\end{aligned}
$$

is the component due to the $k$ th user's signal received at the $m$ th AE. The function $\hat{\psi}(t)=\int_{-\infty}^{\infty} \psi(s) \psi^{*}(s-t) d s$ in (4) represents the chip waveform output of the chip-matched filter.

Let us define the $2 N$-dimensional vectors $\overline{\mathbf{r}}_{m, v}[l]=\left[r_{m, v}[l, 0], \ldots, r_{m, v}[l, 2 N-1]\right]^{T}, \quad \overline{\mathbf{n}}_{m, v}[l]=$ $\left[n_{m, v}[l, 0], \ldots, n_{m, v}[l, 2 N-1]\right]^{T} \quad$ and $\quad \overline{\mathbf{y}}_{k m, v}[l]=$ $\left[y_{k m, v}[l, 0], \ldots, y_{k m, v}[l, 2 N-1]\right]^{T}$. By concatenating these $V M$ vectors corresponding to the $V$ subcarriers and the $M$ AEs, we obtain the following $2 N V M$-dimensional vectors

$\mathbf{r}[l] \quad=\underbrace{[\underbrace{\left.\overline{\mathbf{r}}_{00}^{T}[l], \ldots, \overline{\mathbf{r}}_{(M-1)(V-1)}^{T}[l]\right]^{T}}}_{V M}]_{V M}^{T}, \mathbf{n}[l]$
$[\underbrace{\left.\overline{\mathbf{n}}_{00}^{T}[l], \ldots, \overline{\mathbf{n}}_{(M-1)(V-1)}^{T}[l]\right]^{T}}]^{V}$ and $\mathbf{y}_{k}[l]$ $[\underbrace{\overline{\mathbf{y}}_{k 0,0}^{T}[l], \ldots, \overline{\mathbf{y}}_{k(M-1), V-1}^{T}[l]}_{V M}]^{T}$. Hence, (3) can be rewritten as

$$
\mathbf{r}[l]=\sum_{k=1}^{K} \mathbf{y}_{k}[l]+\mathbf{n}[l] .
$$

Without loss of generality, let $b_{k}[l]$ represent the specific bit of the $k$ th user that falls completely in the $l$ th symbol period and let us normalize the value of $\tau_{k} \in T_{b}$ to the chip duration $T_{c}$. Therefore, we have $\tau_{k}=n_{k} T_{c}$, where $n_{k}$ is an integer between zero and $N-1$. Let $\mathbf{c}_{k}=[c_{k}(0), c_{k}(1), \ldots, c_{k}(N-1), \underbrace{0, \ldots, 0}_{N}]^{T}$ denote a vector of length $2 N$, consisting of the $N$ elements of the spreading sequence of the $k$ th user followed by $N$ zeros. Following the notation in [9], we define $\mathbf{T}_{L}$ and $\mathbf{T}_{R}$ as the acyclic left shift operator and the acyclic right shift operator processing vectors of length $2 N$, respectively. Then, we use $\mathbf{T}_{L}^{n}$ and $\mathbf{T}_{R}^{n}$ to denote $n$ applications of these operators, resulting in $n$ left and right shifts, respectively. Based on these operators, we define $\underline{\mathbf{v}}_{k}^{-1}=\mathbf{T}_{L}^{N-n_{k}} \mathbf{c}_{k}, \underline{\mathbf{v}}_{k}^{0}=\mathbf{T}_{R}^{n_{k}} \mathbf{c}_{k}$ and $\underline{\mathbf{v}}_{k}^{1}=\mathbf{T}_{R}^{N+n_{k}} \mathbf{c}_{k}$.

For each asynchronous user, three consecutive bit intervals overlap with a given observation interval of length $2 T_{b}$. Furthermore, since the system is chip asynchronous, two adjacent chips contribute to each chip sample. The contribution of the $k$ th user $\mathbf{y}_{k}[l]$ to the received vector $\mathbf{r}[l] \in \mathcal{R}^{2 N V M}$ in (5) for the $l$ th symbol is therefore given by

$$
\mathbf{y}_{k}[l]=\check{\mathbf{D}}_{k}\left(b_{k}[l-1] \check{\mathbf{v}}_{k}^{-1}+b_{k}[l] \check{\mathbf{v}}_{k}^{0}+b_{k}[l+1] \check{\mathbf{v}}_{k}^{1}\right),
$$

where $\check{\mathbf{D}}_{k}=\operatorname{diag}\left(\mathbf{D}_{k 0}, \mathbf{D}_{k 1} \ldots, \mathbf{D}_{k(M-1)}\right) \quad$ is a $(2 N V M \times 2 N V M)$-dimensional matrix and $\mathbf{D}_{k m}=\operatorname{diag}\left(A_{k} H_{k m, 0} c_{k, 0}^{\prime}, \ldots, A_{k} H_{k m, 0} c_{k, 0}^{\prime}, \ldots\right.$, $\left.A_{k} H_{k m, V-1} c^{\prime}{ }_{k, V-1}, \ldots, A_{k} H_{k m, V-1} c^{\prime}{ }_{k, V-1}\right)$.

Furthermore, we have $\check{\mathbf{v}}_{k}^{-1}=[\underbrace{\left(\underline{\mathbf{v}}_{k}^{-1}\right)^{T}, \ldots,\left(\underline{\mathbf{v}}_{k}^{-1}\right)^{T}}_{V M}]^{T}, \check{\mathbf{v}}_{k}^{0}=$ $[\underbrace{\left(\underline{\mathbf{v}}_{k}^{0}\right)^{T}, \ldots,\left(\underline{\mathbf{v}}_{k}^{0}\right)^{T}}_{V M}]^{T}$ and $\check{\mathbf{v}}_{k}^{1}=[\underbrace{\left(\underline{\mathbf{v}}_{k}^{1}\right)^{T}, \ldots,\left(\underline{\mathbf{v}}_{k}^{1}\right)^{T}}_{V M}]^{T}$. Upon sub- 
stituting (6) into (5), we arrive at

$$
\begin{aligned}
\mathbf{r}[l]= & \check{\mathbf{D}}_{1} b_{1}[l] \check{\mathbf{v}}_{1}^{0}+\sum_{k=1}^{K} \check{\mathbf{D}}_{k}\left(b_{k}[l-1] \check{\mathbf{v}}_{k}^{-1}+b_{k}[l+1] \check{\mathbf{v}}_{k}^{1}\right) \\
& +\sum_{k=2}^{K} \check{\mathbf{D}}_{k} b_{k}[l] \check{\mathbf{v}}_{k}^{0}+\mathbf{n}[l]
\end{aligned}
$$

and for notational convenience, (7) can be rewritten as

$$
\mathbf{r}[l]=b_{1}[l] \check{\mathbf{p}}_{1}+\sum_{j=2}^{J} b_{j}[l] \check{\mathbf{p}}_{j}+\mathbf{n}[l],
$$

by using the equivalent synchronous model described in [9], where $b_{1}[l]$ is the desired bit and $\check{\mathbf{p}}_{1}=\check{\mathbf{D}}_{1} \check{\mathbf{v}}_{1}^{0}$, while the remaining vectors correspond to the ISI and MAI vectors in (7). Note that we have $2 K \leq$ $J \leq 3 K$.

\section{Blind Channel Estimation}

The eigen-decomposition of the autocorrelation matrix $\mathbf{R}$ of the received MC DS-CDMA signal vector $\mathbf{r}$ is given by [5]

$$
\begin{aligned}
\mathbf{R} & =E\left\{\mathbf{r r}^{H}\right\}=\check{\mathbf{P}} \check{\mathbf{P}}^{H}+\sigma^{2} I_{2 N V M} \\
& =\mathbf{U} \boldsymbol{\Lambda} \mathbf{U}^{H}=\left[\begin{array}{ll}
\mathbf{U}_{s} & \mathbf{U}_{n}
\end{array}\right]\left[\begin{array}{cc}
\boldsymbol{\Lambda}_{s} & 0 \\
0 & \boldsymbol{\Lambda}_{n}
\end{array}\right]\left[\begin{array}{c}
\mathbf{U}_{s}^{H} \\
\mathbf{U}_{n}^{H}
\end{array}\right],
\end{aligned}
$$

where we have $\check{\mathbf{P}}=\left[\check{\mathbf{p}}_{1}, \ldots, \check{\mathbf{p}}_{J}\right], \mathbf{U}=\left[\begin{array}{ll}\mathbf{U}_{s} & \mathbf{U}_{n}\end{array}\right]$ and $\boldsymbol{\Lambda}=$ $\operatorname{diag}\left(\boldsymbol{\Lambda}_{s}, \boldsymbol{\Lambda}_{n}\right)$. Without loss of generality, we assume that the vectors $\check{\mathbf{p}}_{1}, \ldots, \check{\mathbf{p}}_{J}$ are linearly independent and that $J<2 N V M$. Therefore, the matrix $\mathbf{P}$ is a "tall" matrix having a $\operatorname{rank}$ of $\operatorname{rank}(\mathbf{P})=$ $\operatorname{rank}\left(\mathbf{P} \mathbf{P}^{H}\right)=J$ and $\boldsymbol{\Lambda}_{s}=\operatorname{diag}\left\{\lambda_{1}, \ldots, \lambda_{J}\right\}$ contains the largest $J$ eigenvalues of the received signal's autocorrelation matrix $\mathbf{R}$. In contrast to the signal space $\mathbf{U}_{s}=\left[\mathbf{u}_{1}, \ldots, \mathbf{u}_{J}\right]$, the noise space $\mathbf{U}_{n}=\left[\mathbf{u}_{J+1}, \ldots, \mathbf{u}_{2 N V M}\right]$ contains the $(2 N V M-J)$ orthogonal eigenvectors corresponding to the smallest eigenvalues $\sigma^{2}$ in $\boldsymbol{\Lambda}_{n}$.

As shown in [5], [10], the $M L$-dimensional CIR vector $\mathbf{h}_{1}=$ $\left[\mathbf{h}_{10}^{T}, \ldots, \mathbf{h}_{1 m}^{T}, \ldots, \mathbf{h}_{1(M-1)}^{T}\right]$ of the desired user can be estimated by exploiting the orthogonality between the signal subspace and the noise space. More specifically, $\mathbf{U}_{n}$ is orthogonal to the column space of $\check{\mathbf{P}}$ and $\check{\mathbf{p}}_{1}$ represents the column space of $\check{\mathbf{P}}$. Therefore, we have

$$
\mathbf{U}_{n}^{H} \check{\mathbf{p}}_{1}=\mathbf{U}_{n}^{H} \check{\mathbf{V}}_{1}^{0} \check{\mathbf{d}}_{1}=A_{1} \mathbf{U}_{n}^{H} \check{\mathbf{V}}_{1}^{0} \check{\mathbf{C}}^{\prime}{ }_{1} \check{\mathbf{F}}_{L} \mathbf{h}_{1}=0
$$

where we have the $(2 N V M \times V M)$-dimensional TD spreading code matrix $\check{\mathbf{V}}_{1}^{0}=\operatorname{diag}\left(\underline{\mathbf{v}}_{1}^{0}, \ldots, \underline{\mathbf{v}}_{1}^{0}\right)$, the $(V M \times V M)$-dimensional FD spreading code matrix $\check{\mathbf{C}}^{\prime}{ }_{1}=\operatorname{diag}\left(\mathbf{C}^{\prime}{ }_{1}, \ldots, \mathbf{C}^{\prime}{ }_{1}\right)$ and the $(M V \times$ $M L)$-dimensional matrix $\check{\mathbf{F}}_{L}=\operatorname{diag}\left(\mathbf{F}_{L}, \ldots, \mathbf{F}_{L}\right)$.

The estimate $\overline{\mathbf{h}}_{1}$ of the CIR vector $\mathbf{h}_{1}$ can be generated by computing the minimum eigenvector of the matrix $\check{\mathbf{F}}_{L}^{H} \check{\mathbf{C}}^{\prime H}{ }_{1}^{H}\left(\check{\mathbf{V}}_{1}^{0}\right)^{H} \mathbf{U}_{n} \mathbf{U}_{n}^{H} \check{\mathbf{V}}_{1}^{0} \check{\mathbf{C}}^{\prime}{ }_{1} \check{\mathbf{F}}_{L}$. The necessary condition for such an estimate to be unique is that we have $J \leq 2 N V M-L M$. For the worst case scenario of $J=3 K$, a necessary condition imposed on the number of users for the sake of uniquely determining $\mathbf{h}_{1}$ is then $K \leq \frac{(2 N V-L) M}{3}$. When we have $M \geq 2$, the number of users supported can be $K=N V$, provided that the condition of $\frac{(2 M-3) N V}{M} \geq L$ is met, which can be readily satisfied by appropriately choosing $M, N$ and $V$. Compared to the generalized MC DS-CDMA system using a single antenna, the smart antenna aided system is capable of supporting more users, because generally speaking, increasing the number of AEs has the potential of providing a higher degree of freedom and hence it is capable of improving both the achievable system performance and the user capacity. Furthermore, with the aid of an appropriately chosen cyclic prefix, we can remove the phase ambiguity encountered in the channel estimation process by using the techniques proposed in [14].

If the value of $\tau_{1}$ is not available, joint timing and channel estimation must be carried out, which is summarized as follows:

1) Hypothesize a value for $\tau_{1} \in T_{b}$ and construct the matrix $\check{\mathbf{V}}_{1}^{0}\left(\tau_{1}\right)$ based on this hypothesized value;

2) Obtain the best estimate $\overline{\mathbf{h}}_{1}\left(\tau_{1}\right)$ by computing the minimum eigenvector of the matrix $\check{\mathbf{F}}_{L}^{H} \check{\mathbf{C}}^{\prime H}\left(\check{\mathbf{V}}_{1}^{0}\left(\tau_{1}\right)\right)^{H} \mathbf{U}_{n} \mathbf{U}_{n}^{H} \check{\mathbf{V}}_{1}^{0}\left(\tau_{1}\right) \check{\mathbf{C}}^{\prime}{ }_{1} \check{\mathbf{F}}_{L}$;

3) Repeat Steps 1) to 2) for different values of $\tau_{1}$;

4) Attain the best timing estimate $\bar{\tau}_{1}$ by solving the minimization problem of

$$
\bar{\tau}_{1}=\arg \min _{\tau_{1} \in T_{b}} \frac{\left\|\mathbf{U}_{n}^{H} \check{\mathbf{V}}_{1}^{0}\left(\tau_{1}\right) \check{\mathbf{C}}^{\prime}{ }_{1} \check{\mathbf{F}}_{L} \overline{\mathbf{h}}_{1}\left(\tau_{1}\right)\right\|^{2}}{\left\|\check{\mathbf{V}}_{1}^{0}\left(\tau_{1}\right) \check{\mathbf{C}}^{\prime}{ }_{1} \check{\mathbf{F}}_{L} \overline{\mathbf{h}}_{1}\left(\tau_{1}\right)\right\|^{2}} ;
$$

5) Arrive at the best estimate $\overline{\mathbf{h}}_{1}\left(\bar{\tau}_{1}\right)$ based on $\bar{\tau}_{1}$.

In practice, we have to quantize the infinite number of possible hypothesized values of $\tau_{1}$ in the interval $\left[0, T_{b}\right]$ to a finite set. Since the optimization problem in (11) is one-dimensional, not all choices of the pair $\left(\tau_{1}, \mathbf{h}_{1}\right)$ are legitimate. The procedure described above leads to the best estimate $\overline{\mathbf{h}}_{1}\left(\tau_{1}\right)$ for a specific hypothesized value of $\tau_{1}$. Therefore, the search space is substantially reduced, rendering the above method more practical. Furthermore, since $\Omega\left(\tau_{1}\right)=$ $\frac{\left\|\mathbf{U}_{n}^{H} \mathbf{V}_{1}^{0}\left(\tau_{1}\right) \mathbf{C}^{\prime}{ }_{1} \mathbf{F}_{L} \overline{\mathbf{h}}_{1}\left(\tau_{1}\right)\right\|^{2}}{\left\|\mathbf{V}_{1}^{0}\left(\tau_{1}\right) \mathbf{C}^{\prime}{ }_{1} \mathbf{F}_{L} \overline{\mathbf{h}}_{1}\left(\tau_{1}\right)\right\|^{2}}$ is a continuous function of $\tau_{1}$, it must have a minimum within the closed interval $\left[0, T_{b}\right]$, which hence guarantees the optimal choice of $\tau_{1}$ that minimizes the cost function, provided that we search on a sufficiently fine grid.

\section{Blind And Group-Blind Multiuser Detection}

Given the prior knowledge of the desired user's signature waveform, the linear MMSE MUD weights $\mathbf{w}_{1}=\mathbf{m}_{1}$ can be expressed in terms of the signal subspace components $\mathbf{U}_{s}, \boldsymbol{\Lambda}_{s}, \sigma^{2}$ and the estimated composite signature waveform $\overline{\mathbf{p}}_{1}=\check{\mathbf{V}}_{1}^{0}\left(\tau_{1}\right) \check{\mathbf{C}}_{1}^{\prime} \check{\mathbf{F}}_{L} \overline{\mathbf{h}}_{1}$ as

$$
\mathbf{m}_{1}=\mathbf{U}_{s} \Lambda_{s}^{-1} \mathbf{U}_{s}^{H} \overline{\mathbf{p}}_{1},
$$

which is obtained by solving the following optimization problem [5]

$$
\mathbf{m}_{1}=\arg \min _{\mathbf{w} \in \mathcal{R}^{2 N V M}} E\left\{\left|b_{1}-\mathbf{w}^{H} \mathbf{r}\right|^{2}\right\}=\mathbf{R}^{-1} \overline{\mathbf{p}}_{1} .
$$

Furthermore, the so-called form-II linear hybrid group-blind MUD $\overline{\mathbf{w}}_{1}$ [5], which exploits the knowledge of a group of $\tilde{K}$ composite signature waveforms corresponding to $\tilde{K}(\tilde{K} \leq K)$ intracell users, is given by the solution of the following constrained optimization problem [5]

$$
\overline{\mathbf{w}}_{1}=\arg \min _{\mathbf{w}_{1} \in \operatorname{range}(\mathbf{P})} E\left\{\left|b_{1}-\mathbf{w}_{1}^{H} \mathbf{r}\right|^{2}\right\}
$$

subject to $\mathbf{w}_{1}^{H} \tilde{\mathbf{P}}=\mathbf{1}_{1}^{T}$, where $\mathbf{1}_{1}$ is a $\tilde{J}$-dimensional vector having all-zero elements, except for the 1st element, which is one, and $\tilde{\mathbf{P}}=\left[\overline{\mathbf{p}}_{1}, \ldots, \overline{\mathbf{p}}_{\tilde{J}}\right]$ is a $(2 N V M \times \tilde{J})$-dimensional matrix constructed based on the estimated CIRs, on the timing information and on the TFDomain spreading signature waveforms of all the $\tilde{K}$ intracell users. Hence we have $2 \tilde{K} \leq \tilde{J} \leq 3 \tilde{K}$. Using the method of Lagrange multipliers [5] in (14), we obtain the form-II linear hybrid group-blind MUD's weight vector in the form of [5]

$$
\overline{\mathbf{w}}_{1}=\mathbf{U}_{s} \boldsymbol{\Lambda}_{s}^{-1} \mathbf{U}_{s}^{H} \tilde{\mathbf{P}}\left(\tilde{\mathbf{P}}^{H} \mathbf{U}_{s} \boldsymbol{\Lambda}_{s}^{-1} \mathbf{U}_{s}^{H} \tilde{\mathbf{P}}\right)^{-1} \mathbf{1}_{1} .
$$


For the group-blind MUD of (15), the interfering signals arriving from 'known' intracell users are nulled by a projection of the received signal onto the orthogonal subspace of these users' signal subspace. The unknown interfering users' signals inflicted by other-cell users are suppressed by identifying the subspace spanned by these intercell users, followed by a linear transformation in this subspace based on the MMSE criterion.

\section{Performance Results}

All investigations of this section were based on evaluating the performance of the subspace-based blind and group-blind MUDs employed in the uplink of a smart antenna aided generalized MC DSCDMA system, when communicating over a dispersive ST Rayleigh fading channel contaminated by AWGN. An antenna array having $M$ number of AEs is considered in our investigations. When the AEs are located sufficiently far apart, the MC DS-CDMA signal experiences independent fading upon reaching the different AEs, hence we have the cross-correlation coefficient $\rho=0$ among the AEs. By contrast, when the AEs are separated by a distance of half a wavelength, we have $\rho=1$, which implies that these AEs are fully correlated. The basic parameters of the blind and group-blind MUDs invoked for the generalized MC DS-CDMA system considered are summarized in Table I.

\begin{tabular}{|l|l|}
\hline Parameters & Value \\
\hline Chips-spaced CIR length $L$ & 3 \\
\hline Normalized Dopper frequency & 0.01 \\
\hline Short cyclic prefix & 2 chips \\
\hline Burst length $M_{F}$ & 256 \\
\hline Number of subcarriers $V$ & 4 \\
\hline FD Spreading gain & 4 \\
\hline TD Spreading gain & 15 \\
\hline
\end{tabular}

TABLE I

BASIC SIMULATION PARAMETERS FOR THE BLIND AND GROUP-BLIND MUDS INVOKED FOR THE GENERALIZED MC DS-CDMA UPLINK.

In Figure 2(a), three different types of antenna arrays, namely a single antenna as well as an array having $M=2$ correlated AEs or $M=2$ uncorrelated AEs were employed. The simulation results showed that the group-blind MUD benefitting from the knowledge of more intracell users' signature waveforms attained a better BER performance. Furthermore, Figure 2(a) demonstrated that as expected, the blind MUD operating in conjunction with an antenna array having $M=2$ uncorrelated AEs achieved a better BER performance than that having $M=2$ correlated AEs. The blind MUD employing a single antenna attained the worst BER performance. In Figure 2(b) we observe that the blind and group-blind space-time MUDs equipped with the subspace based CIR estimator perform only slightly worse than the estimator exploiting the perfect knowledge of the CIRs, which implies that the associated performance degradation imposed by the channel estimator error is negligible.

In Figure 3(a), an antenna array having $M=2$ uncorrelated AEs was employed and we had $\rho=0$, where the BER performance of the blind and the group-blind MUDs was presented as a function of the number of users supported. Note that the system having $V=4$, $N=15$ and hence capable of serving $K=60$ users was full loaded. Finally, Figure 3(b) characterizes the SNR gains achieved by increasing the number of AEs, demonstrating that increasing the number of AEs is capable of providing an increased degree of freedom and hence

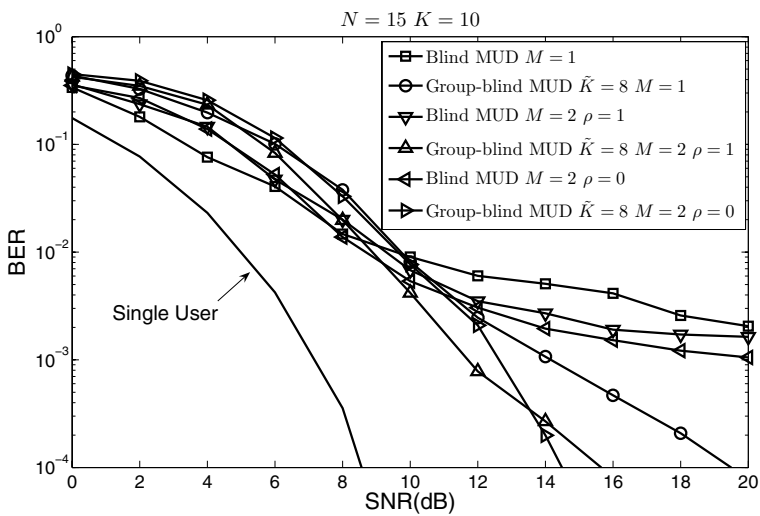

(a)

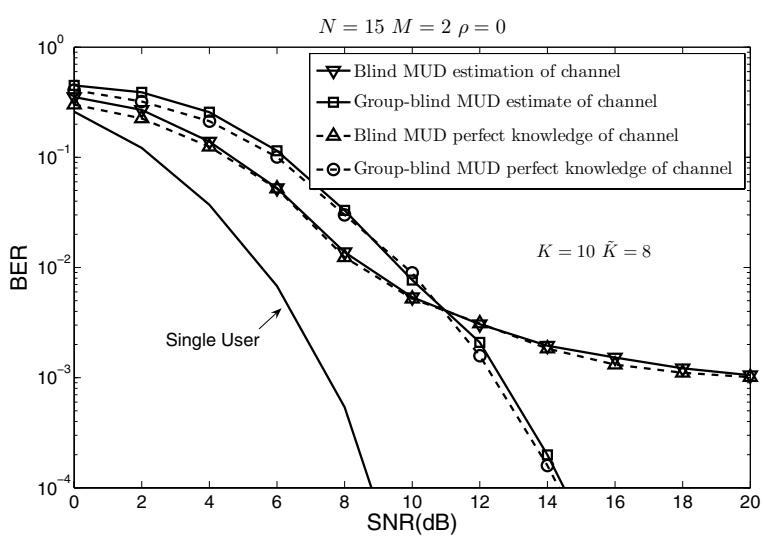

(b)

Fig. 2. BER performance of the various MC DS-CDMA MUDs considered A total of $K=10$ users were supported and the group-blind MUDs benefitted from the knowledge of $\tilde{K}=8$ intracell users' spreading codes. The remaining system parameters are summarized in Table I. (a) In this figure, three different types of antenna arrays were studied, namely a single antenna as well as an antenna array having $M=2$ correlated and uncorrelated AEs. (b) In this figure, an antenna array having $M=2$ uncorrelated AEs was employed and we had $\rho=0$. Both the scenario of having the perfect knowledge of the complex-valued CIRs and their realistic estimation were considered.

of substantially improving the attainable system performance at the expense of a higher system complexity.

\section{CONCLUSIONS}

In this contribution subspace-based blind and group-blind spacetime MUDs were investigated in the context of smart antenna aided generalized MC DS-CDMA systems. As expected, the group-blind MUD benefitting from the knowledge of more intracell users' signature waveforms attained a better BER performance. The blind and group-blind space-time MUDs equipped with the subspace based CIRs estimator perform only slightly worse than the estimator exploiting the perfect knowledge of the CIRs, which implied that the associated performance degradation imposed by the channel estimator error is negligible. Furthermore, our simulation results suggested that increasing the number of AEs is capable of providing an increased degree of freedom and hence of substantially improving the attainable performance at the expense of a higher system complexity. 


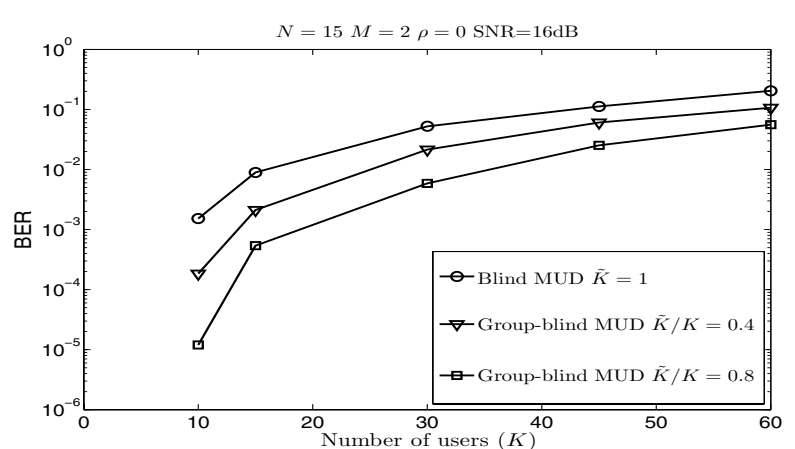

(a)

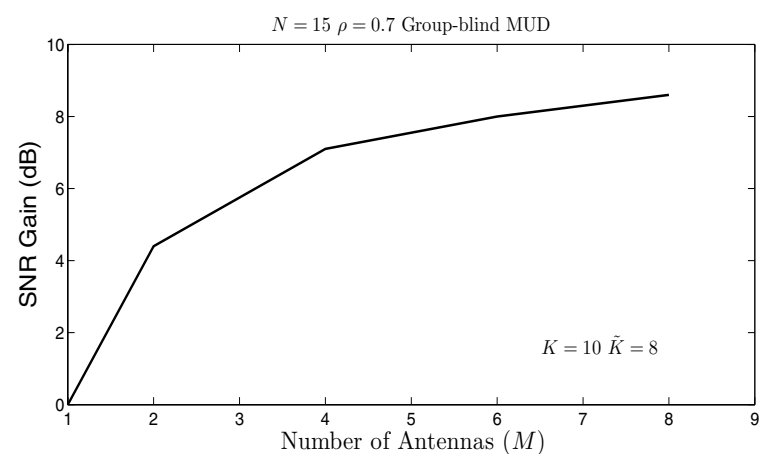

(b)

Fig. 3. (a) BER versus the number of users, $K$, performance of the various MC DS-CDMA MUDs considered. An antenna array having $M=2$ uncorrelated AEs was employed and we had $\rho=0$. The SNR was fixed to $16 \mathrm{~dB}$. When considering the group-blind MUDs, the ratio of $\tilde{K} / K$ was assigned to 0.4 or 0.8 . (b) SNR gain versus the number of the receive antennas, $M$, performance of group-blind MUDs at a BER of $10^{-4}$. Antenna arrays having a cross-correlation coefficient of $\rho=0.7$ were used. A total of $K=10$ users were supported and the group-blind MUDs benefitted from the knowledge of $\tilde{K}=8$ intracell users' spreading codes. The remaining system parameters are summarized in Table I.

\section{REFERENCES}

[1] L. Hanzo, L. L. Yang, E. L. Kuan, and K. Yen, Single- and Multi- Carrier DS-CDMA. John Wiley \& Sons - IEEE Press, 2003, 1060 pages.

[2] S. Verdu, Multiuser Detection. Cambridge University Press, 1998, 474 pages.

[3] M. Honig and U. Madhow and H. V. Poor, "Blind Adaptive Multiuser Detection," IEEE Transactions on Information Theory, vol. 41, pp. 944 960, July 1995.

[4] X. Wang and H. V. Poor, "Blind Multiuser Detection: A Subspace Approach," IEEE Transactions on Information Theory, vol. 44, pp. 677-690, March 1998.

[5] X. Wang and A. Host-Madsen, "Group-Blind Multiuser Detection for Uplink CDMA," IEEE Journal on Selected Areas in Communications, vol. 17, pp. 1971-1984, Nov. 1999.

[6] R. Lupas and S. Verdú, "Linear Mulituser Detectors for Synchronous Code Division Multiple Access Channel," IEEE Transactions on Communications, vol. 35, pp. 123-136, Jan. 1989.

[7] D. S. Chen and S. Roy, "An Adaptive Multiuser Receiver for CDMA Systems," IEEE Journal on Selected Areas in Communications, vol. 12, pp. 808-816, June 1994

[8] G. Woodward and B. S. Vucetic, "Adaptive detection for DS-CDMA," Proceedings of the IEEE, vol. 86, pp. 1413-1434, July 1998.

[9] U. Madhow, "MMSE interference suppression for timing acquisition and demodulation in direct-sequence CDMA systems," IEEE Transactions on Communications, vol. 46, pp. 1065-1075, Aug. 1998.

[10] X. Wang and H. V. Poor, "Blind Equalization and Multiuser Detection in Dispersive CDMA Channels," IEEE Transactions on Communications, vol. 6, pp. 91-103, Jan. 1998.

[11] J. Namgoong and T. F. Wong and J. S. Lehnert, "Subspace multiuser detection for multicarrier DS-CDMA," IEEE Transactions on Communications, vol. 48, pp. 1897-1908, Nov. 2000.

[12] L.-L. Yang and L. Hanzo, "Performance of generalized multicarrier DSCDMA over Nakagami- $m$ fading channels," IEEE Transactions on Communications, vol. 50, pp. 956 - 966, June 2002.

[13] L. Hanzo, M. Munster, B. J. Choi, and T. Keller, OFDM and MC-CDMA. John Wiley \& Sons - IEEE Press, 2003, 960 pages.

[14] S. Zhou and B. Muquet and G. B. Giannakis, "Subspace-Based (Semi-) Blind Channel Estimation for Block Precoded Space-Time OFDM," IEEE Transactions on Signal Processing, vol. 50, pp. 1215-1228, May 2002. 